\title{
Long-term monitoring after surgical ablation for atrial fibrillation: How much is enough?
}

\author{
James R. Edgerton, MD, ${ }^{\text {a,b }}$ Cecile Mahoney, BS, ${ }^{\mathrm{b}}$ Michael J. Mack, MD, ${ }^{\mathrm{a}, \mathrm{b}}$ Karen Roper, PhD, ${ }^{\mathrm{b}}$ and \\ Morley A. Herbert, $\mathrm{PhD}^{\mathrm{c}}$
}

\begin{abstract}
Introduction: Evaluating and comparing the success of surgical ablation techniques in the treatment of atrial fibrillation is complicated by clinicians' use of varying techniques to measure the burden of atrial fibrillation after ablation. Intuitively, one would expect longer monitoring to be more accurate, picking up atrial fibrillation events occurring at a low rate, but how long is long enough? This study compared rates of normal sinus rhythm recorded after atrial fibrillation ablation in a cohort of patients monitored for a range of durations.
\end{abstract}

\begin{abstract}
Methods: Two hundred fifty-four patients (50.4\% paroxysmal) underwent surgical ablation for treatment of atrial fibrillation. All patients were monitored at 6 months with both electrocardiography and either an event monitor or implanted pacemaker device that could be interrogated. Event monitoring and pacemaker data were analyzed for rhythm at 24 hours, 7 days, and 14 days; pacemaker data were also analyzed at 3 months.

Results: In the overall group, we found that rates of normal sinus rhythm detected were greatest with electrocardiography $(91.7 \%)$ and decreased significantly at each of the longer durations $(88.2 \%$ at 24 hours, $82.7 \%$ at 7 days, $81.1 \%$ at 14 days). Pacemaker data from a subset of patients revealed minimal or no statistically significant changes from 7 days to 3 months. Results were consistent across types of atrial fibrillation.
\end{abstract}

Conclusions: These findings suggest that rhythm measurements in patients with atrial fibrillation differ according to the measurement duration used. We recommend longer-term monitoring, with 7 days providing both good accuracy and good patient compliance. (J Thorac Cardiovasc Surg 2011;142:162-5)

Atrial fibrillation (AF) is a common condition that affects approximately 2 million people in the United States and is increasing in prevalence as the population ages. ${ }^{1}$ During the last several decades, catheter and surgical ablation techniques for the treatment of $\mathrm{AF}$ have evolved rapidly, but a consistent definition of successful ablation and the clinical end points that constitute a successful ablation have been lacking, as has an analysis of the various methods used to monitor success.

In 2003, the US Food and Drug Administration recommended "freedom from symptomatic atrial fibrillation at one year" in end point analysis of clinical studies on catheter ablation of $\mathrm{AF}$ (www.fda.gov/cdrh/ode/guidance/1229. pdf). Monitoring for symptoms in patients with AF is both unreliable, because $50 \%$ of recurrences occur in patients without symptoms, ${ }^{2}$ and inaccurate, because symptoms are over reported, ${ }^{3}$ with only $52 \%$ of patient symptoms correlated with documented measures of $\mathrm{AF}^{4-7}$

From The Heart Hospital Baylor Plano, ${ }^{\text {a Plano, Tex; the Cardiopulmonary Research }}$ Science and Technology Institute, ${ }^{\mathrm{b}}$ Dallas, Tex; and Medical City Dallas Hospital, ${ }^{\mathrm{c}}$ Dallas, Tex.

Disclosures: J.R.E. is a paid consultant with AtriCure, Inc, West Chester, Ohio.

Received for publication Sept 17, 2010; revisions received Dec 22, 2010; accepted for publication Jan 24, 2011; available ahead of print March 7, 2011.

Address for reprints: James R. Edgerton, MD, Cardiovascular Specialty Association of North Texas PA, 4708 Alliance Blvd, Suite 700, Plano, TX 75093 (E-mail: edgertonjr@aol.com).

0022-5223/\$36.00

Copyright (c) 2011 by The American Association for Thoracic Surgery doi:10.1016/j.jtcvs.2011.01.050
The Heart Rhythm Society Task Force produced a consensus statement that is the current standard, defining an occurrence as any episode of AF, flutter, or tachycardia with a duration of 30 seconds or longer. ${ }^{8}$ Even though some research suggests that such 30 -second episodes are relatively common, ${ }^{9}$ the consensus statement does provide the currently accepted and agreed definition of success.

Another problem is defining acceptable monitoring and surveillance standards for AF. The Task Force recommends 24-hour Holter monitoring as an acceptable minimal monitoring strategy for patients in a clinical trial. The consensus statement does not, however, justify why the specific $24-$ hour monitor duration was recommended. In comparison studies, "snapshot" (30-second) and 24-hour electrocardiograms (ECGs) with 7-day event recording ${ }^{10.11}$; ECG with 5-day monitoring ${ }^{12}$; and 24-hour, 7-day, and 30-day simulated intermittent monitoring with continuous pacemaker readings ${ }^{13}$ have all been shown to have poor agreement. Piorkowski and colleagues ${ }^{14}$ have argued that continuous monitoring is superior to snapshot monitoring because of the risk of missing asymptomatic episodes.

Despite these obvious discrepancies, no study to date has compared the results of different monitoring durations in the same patient population. The objective of this study was to compare the accuracy of snapshot ECG with those of various intervals of long-term monitoring for patients who had undergone surgical ablation for the treatment of paroxysmal, persistent, or longstanding persistent AF. 


\section{Abbreviations and Acronyms \\ $\mathrm{AF}=$ atrial fibrillation \\ $\mathrm{ECG}=$ electrocardiogram \\ $\mathrm{NSR}=$ normal sinus rhythm}

\section{MATERIALS AND METHODS}

Data were extracted from our Society of Thoracic Surgeons customized AF database for all patients who (1) underwent surgical ablation between January 2004 and June 2009, (2) had at least 6 months of follow-up, and (3) had both a snapshot ECG and long-term monitoring (14-day event monitor, or pacemaker interrogation) at 6 months after the AF ablation. The event monitors were a mixture of devices provided by Medicomp (Medicomp, Inc, Melbourne, Fla) or CardioNet (Conshohocken, Pa). These were autocapture devices that required no activation by the patient.

Patients undergoing ablation either stand-alone or with concomitant procedures were eligible for the study. A total of 254 patients met the criteria. The ablations were carried out with a variety of energy sources, including cryothermia, focused ultrasound, microwave, and bipolar or unipolar radiofrequency.

The study was approved by the North Texas Institutional Review Board at Medical City with an exemption of consent.

Starting at the time of their 6-month office follow-up visit, all patients were monitored for 14 days with an event monitor. Data were collected and then used to analyze the rhythm in the first 24 hours, first 7 days, and full 14 days of monitoring. Patients with pacemakers also provided data for the whole 3-month period until next reset. Office ECGs were performed at the visit.

All data were analyzed with SAS version 9.2 statistical software (SAS Institute, Inc, Cary, NC). Means, SDs, and medians were calculated for continuous variables. Categoric variables were analyzed with $\chi^{2}$ statistics. The comparisons of the different monitoring periods were subjected to the McNemar test for discordant pairs. The McNemar test is a $\chi^{2}$ test for within-subject populations; in this case, the same patients were being measured by multiple methods.

\section{RESULTS}

The demographic characteristics, types of AF, ablation procedures, and energy sources used are shown in Table 1.

Figure 1 shows the percentages of patients who were recorded as being in normal sinus rhythm (NSR) at each of the monitoring periods. As expected, increasing the monitoring period detected more of the infrequent occurrences of AF. Statistical comparisons of the numbers of patients recorded as being in NSR showed statistically significant changes as the period of monitoring was increased from snapshot ECG to 14 days. The change achieved by monitoring past 7 days was small $(1.6 \%)$, however, suggesting little improvement in accuracy with the extra time.

Subset analysis was carried out on data from 56 patients who had implanted pacemakers allowing data recording for 3 -month periods. Figure 2 shows the NSR percentages out to 3 months of monitoring in that group of patients. These data showed similar decreases in the percentage of patients reported as being in NSR as the monitoring period increased. In the pacemaker group, analysis shows that after
TABLE 1. Patient demographic and operative characteristics

\begin{tabular}{lc}
\hline \multicolumn{1}{c}{ Characteristic } & Value \\
\hline No. of patients & 254 \\
Age $(\mathrm{y})$ & \\
$\quad$ Mean \pm SD & $64.9 \pm 11.4$ \\
Median & 65.0 \\
Male (\%) & $61.0(155 / 254)$ \\
Atrial fibrillation $>12$ mo (\%) & $89.8(176 / 196)$ \\
Stand-alone ablation $(\%)$ & $42.5(108 / 254)$ \\
On-pump procedure $(\%)$ & $54.8(138 / 252)$ \\
Energy source (\%) & \\
Radiofrequency & $61.8(155 / 251)$ \\
Cryothermia & $20.7(52 / 251)$ \\
High-intensity focused ultrasound & $14.7(37 / 251)$ \\
Microwave & $2.8(7 / 251)$ \\
Type of atrial fibrillation before ablation $(\%)$ & \\
Paroxysmal & $50.4(128 / 254)$ \\
Persistent & $13.8(35 / 254)$ \\
Long-standing persistent & $35.8(91 / 254)$ \\
Pulmonary vein isolation procedure (\%) & $100(254 / 254)$ \\
Left atrial appendage removed (\%) & $85.0(142 / 167)$ \\
Left atrial size (cm) & \\
Mean \pm SD & $4.9 \pm 1.1$ \\
Median & 4.9 \\
Ejection fraction (\%) & \\
Mean \pm SD & $50.6 \pm 12.7$ \\
Median & 50.0 \\
\hline
\end{tabular}

7 days, there was a minimal change in the number of patients reported as being in NSR. The 14-day data detected the same patients as at 7 days; extending the patient monitoring to 3 months did pick up a few more cases of AF, but there was no statistically significant difference relative to either 7 or 14 days.

Figure 3 presents the results when looking only at patients who had paroxysmal AF at the time of ablation. Further monitoring past 7 days picked up a small number of new patients with continuing AF.

Figure 4 reports the data for 126 patients with persistent or long-standing persistent AF. The data again show that monitoring for longer than 7 days turned up few extra cases.

Subset analysis of the data captured from 24 patients with paroxysmal AF who also had implanted pacemakers showed $75.0 \%$ of them to be in NSR at 7 and 14 days of monitoring, whereas the pacemaker data from 3 months showed the rate slightly decreased, to $79.8 \%(P=.317)$.

Among the 32 patients with pacemakers, all of whom originally had the diagnosis of either persistent or longstanding persistent $\mathrm{AF}$, the pacemaker data showed equal rates of $78.1 \%$ at 7 and 14 days, with a decrease to $71.9 \%$ at 3 months $(P=.157)$.

\section{DISCUSSION}

The objective of this study was to determine the accuracies of various durations of monitoring needed to confirm 


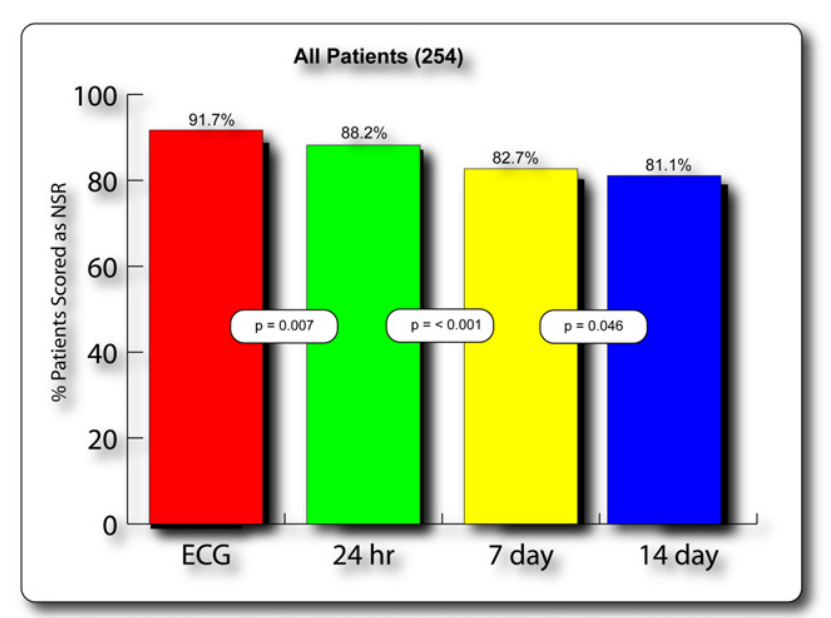

FIGURE 1. Percentages of all patients scored as being in normal sinus rhythm $(N S R)$ at differing monitoring durations. ECG, In-office snapshot electrocardiogram.

postablation success in patients who had undergone surgical ablation for the treatment of AF. These patients had widely differing operative techniques, but neither the technique nor the absolute success rate is germane to this study. Accurate identification of patients with recurrent AF is critical, because such patients may need to undergo further treatment, including anticoagulation therapy to reduce the risk of stroke. $^{13,15}$

Consistent with the results of previous research, ${ }^{10-12}$ our data show that snapshot ECG underestimates the true rate of AF episodes after ablation. In-office snapshot ECG resulted in the highest rates of ablation success when compared with, 24-hour, 7-day, and 14-day monitoring in all cases. Monitoring for longer than 7 days increased the accuracy slightly, but the change relative to the 7 days data was generally not significant.

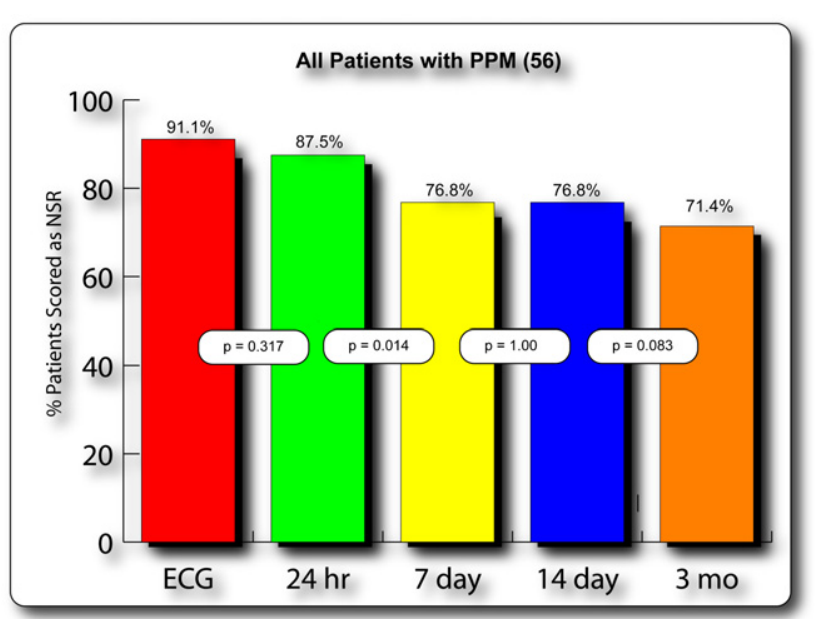

FIGURE 2. Percentages of patients with implanted pacemakers (PPM) scored as being in normal sinus rhythm (NSR) at differing monitoring durations. ECG, In-office snapshot electrocardiogram.



FIGURE 3. Percentages of all patients with preoperative paroxysmal atrial fibrillation scored as being in normal sinus rhythm $(N S R)$ at differing monitoring durations. $E C G$, In-office snapshot electrocardiogram.

Monitoring for 3 months (only practical for patients with implanted pacemakers) did not increase accuracy relative to monitoring for 2 weeks, detecting few extra patients with AF. This finding suggests that prolonged monitoring with either external or implantable devices is not necessary.

When patient subgroups are analyzed according to preoperative type of AF, there was no change in the results. Although one might expect that patients with long-standing persistent $\mathrm{AF}$ would require shorter monitoring periods to determine failure, this is not true. The explanation lies in the fact that when these patients have failure of ablation, the new disorder most often is paroxysmal AF, rather than persistent AF.

These findings call into question the recommendation of the Heart Rhythm Society Task Force on Catheter and Surgical Ablation of Atrial Fibrillation, which states that for

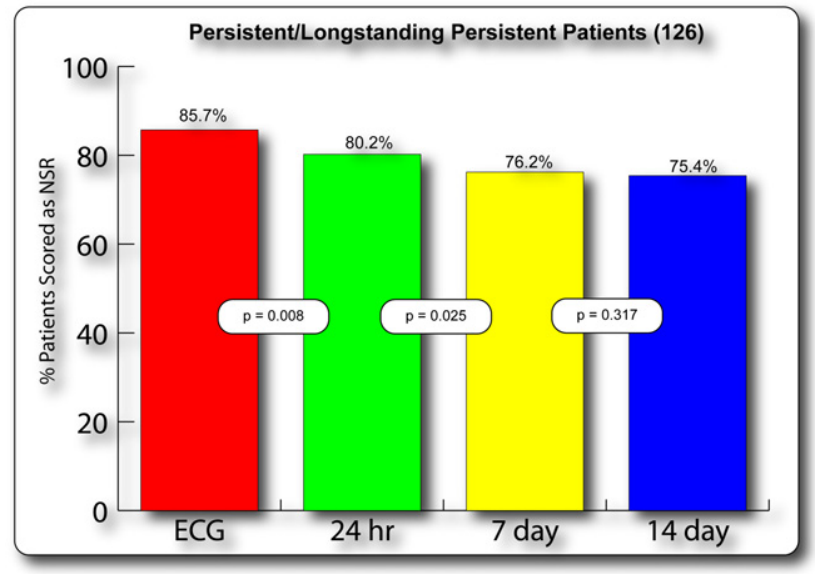

FIGURE 4. Percentages of all patients with preoperative persistent or long-standing persistent atrial fibrillation scored as being in normal sinus rhythm (NSR) at differing monitoring durations. ECG, In-office snapshot electrocardiogram. 
patients in a clinical trial, 24-hour Holter monitoring should be considered an acceptable minimal monitoring strategy. ${ }^{8}$ The percentage of patients reported as being in NSR was found to be significantly higher with 24-hour monitoring than with any of the longer monitoring intervals measured here (7 days, 14 days, or 3 months). Our findings thus suggest that clinical studies and treatment strategies that rely on 24-hour monitoring are likely to overestimate freedom from AF recurrence. ${ }^{11,13}$

Among the patients in this study, 7-day monitoring provided a more accurate determination of ablation success than did 24-hour monitoring. The 7-day monitoring period consistently differed from the shorter capture durations and yet did not differ substantially from the longer intervals studied. Furthermore, patient compliance with use of external devices tends to decrease as the monitoring period increases, ${ }^{5}$ and cost charges for external devices are frequently based on the length of time the device is in use by the patient. Economic considerations such as this may be damaging to a patient's willingness to undergo long periods of monitoring. This suggests that 7-day monitoring provides the best compromise for achieving accurate and consistent outcomes measurement in patients with $\mathrm{AF}$ who have undergone surgical ablation. ${ }^{14}$

\section{Study Limitations}

The major limitation of the study is that the event monitors used measured any AF that lasted longer than $15 \mathrm{sec}-$ onds as an event. Given that the Heart Rhythm Society guidelines call for events to last at least 30 seconds, these event monitors will have overreported by an unknown amount. Pacemakers, in contrast, accurately reported the durations of all events.

\section{CONCLUSIONS}

Our findings demonstrate the potential inaccuracy of both snapshot ECG and 24-hour monitoring to detect AF recurrence in patients who have undergone surgical ablation, despite the recommendation of the shorter 24-hour Holter monitor in the Task Force guidelines. Future research is required to support the development of a consistent definition of ablation success. It is clear from this study, however, that developed guidelines should incorporate longer monitoring durations, with 7 days the likely best compromise. It is appropriate to reexamine the recommendations of the consensus statement. Future published studies examining the success of catheter or surgical ablation should monitor patients for a minimum of 7 days.

\section{References}

1. Arya A, Piorkowski C, Sommer P, Kottkamp H, Hindricks G. Clinical implications of various follow up strategies after catheter ablation of atrial fibrillation. Pacing Clin Electrophysiol. 2007;30:458-62.

2. Cheung JW, Keating RJ, Stein KM, Markowitz SM, Iwai S, Shah BK, et al. Newly detected atrial fibrillation following dual chamber pacemaker implantation. J Cardiovasc Electrophysiol. 2006;17:1323-8.

3. Hindricks G, Piorkowski C, Tanner H, Kobza R, Gerds-Li JH, Carbucicchio C, et al. Perception of atrial fibrillation before and after radiofrequency catheter ablation: relevance of asymptomatic arrhythmia recurrence. Circulation. 2005; 112:307-13

4. Cheema A, Dong J, Dalal D, Marine JE, Henrikson CA, Spragg D, et al. Circumferential ablation with pulmonary vein isolation in permanent atrial fibrillation. Am J Cardiol. 2007;99:1425-8.

5. Vasamreddy CR, Dalal D, Dong J, Cheng A, Spragg D, Lamiy SZ, et al Symptomatic and asymptomatic atrial fibrillation in patients undergoing radiofrequency catheter ablation. J Cardiovasc Electrophysiol. 2006;17:134-9.

6. Gaynor SL, Schuessler RB, Bailey MS, Ishii Y, Boineau JP, Gleva MJ, et al. Surgical treatment of atrial fibrillation: predictors of late recurrence. $J$ Thorac Cardiovasc Surg. 2005;129:104-11.

7. Voeller RK, Bailey MS, Zierer A, Lall SC, Sakamoto S, Aubuchon K, et al. Isolating the entire posterior left atrium improves surgical outcomes after the Cox maze procedure. J Thorac Cardiovasc Surg. 2008;135:870-7.

8. European Heart Rhythm Association (EHRA), European Cardiac Arrhythmia Society (ECAS), American College of Cardiology (ACC), American Heart Association (AHA), Society of Thoracic Surgeons (STS)Calkins H. HRS/EHRA/ ECAS expert Consensus Statement on catheter and surgical ablation of atrial fibrillation: recommendations for personnel, policy, procedures and follow-up. A report of the Heart Rhythm Society (HRS) Task Force on catheter and surgical ablation of atrial fibrillation. Heart Rhythm. 2007;4:816-61.

9. Orlov MV, Ghali JK, Araghi-Niknam M, Sherfesee L, Sahr D, Hettrick DA, et al. Asymptomatic atrial fibrillation in pacemaker recipients: incidence, progression, and determinants based on the atrial high rate trial. Pacing Clin Electrophysiol. 2007;30:404-11.

10. Puskas JD, Corvera JS, Neill A, Kilgo P, Vassiliades T, Guyton RA. "Spot' ECGs underestimate atrial fibrillation recurrence after surgical ablation. Innovations. 2008;3:7-11.

11. Kottkamp H, Tanner H, Kobza R, Schirdewahn P, Dorszewski A, Gerds-Li JH, et al. Time courses and quantitative analysis of atrial fibrillation episode number and duration after circular plus linear left atrial lesions: trigger elimination or substrate modification: early or delayed cure? J Am Coll Cardiol. 2004;44: 869-77.

12. Ad N, Henry L, Hunt S, Barnett S, Stone L. The Cox-Maze III procedure success rate: comparison by electrocardiogram, 24-hour Holter monitoring and longterm monitoring. Ann Thorac Surg. 2009;88:101-5.

13. Ziegler PD, Koehler JL, Mehra R. Comparison of continuous versus intermittent monitoring of atrial arrhythmias. Heart Rhythm. 2006;3:1445-52.

14. Piorkowski C, Kottkamp H, Tanner H, Kobza R, Nielsen JC, Arya A, et al. Value of different follow-up strategies to assess the efficacy of circumferential pulmonary vein ablation for the curative treatment of atrial fibrillation. J Cardiovasc Electrophysiol. 2005;16:1286-92.

15. Oral H, Pappone C, Chugh A, Good E, Bogun F, Pelosi F Jr, et al. Circumferential pulmonary-vein ablation for chronic atrial fibrillation. $N$ Engl J Med. 2006;354: 934-41. 